\title{
Evidence for neuronal localisation of enteroviral sequences in motor neurone disease/amyotrophic lateral sclerosis by in situ hybridization
}

\author{
C.J. Woodall, D.I. Graham*
}

School of Life Sciences, GCU, Glasgow, UK and *University of Glasgow, Department of Neuropathology, Institute of Neurological Sciences, Southern General Hospital, Glasgow, UK

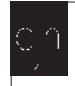

(C)2004, European Journal of Histochemistry

Sequences resembling those of human enterovirus type B sequences have been associated with motor neurone disease/amyotrophic lateral sclerosis. In a previous study we detected enteroviral sequences in spinal cord/brain stem from cases of motor neurone disease/amyotrophic lateral sclerosis, but not controls. Adjacent tissue sections to two of those strongly positive for these sequences by reverse-transcriptase polymerase chain reaction were analyzed by in situ hybridization with digoxigenin-labelled virus-specific antisense riboprobes. In one case, a female aged 83 showing 12 month rapid progressive disease, signal was specifically localized to cells identifiable as motor neurones of the anterior horn. In another case, a male aged 63 with a 60-month history of progressive muscle weakness, dysarthia, dyspnoea and increased tendon reflexes, signal was located to neurones in the gracile/cuneate nuclei of the brain stem tissue block that had been analyzed. This case showed loss of neurones in the anterior horn of the spinal cord by histopathologic examination which would account for clinical signs of motor neurone disease/amyotrophic lateral sclerosis. Dysfunction of the gracile/cuneate nuclei might have been masked by the paralytic disease. These structures are adjacent to the hypoglossal nuclei, and suggest either localised dissemination from hypoglossal nuclei or a possible route of dissemination of infection through the brainstem to the hypoglossal nuclei. These findings provide further evidence for the possible involvement of enteroviruses in motor neurone disease/amyotrophic lateral sclerosis.

Key words: enterovirus; echovirus; in situ hybridization; motor neurone disease; amyotrophic lateral sclerosis.

Correspondence: Christopher J. Woodall, School of Life Sciences, GCU, Cowcaddens Road, Glasgow G4 OBA, UK. Phone: international +44-141-331-8245, Fax: international +44-141-331-3208. E-mail: c.woodall@gcal.ac.uk

Paper accepted on November 14, 2003

European Journal of Histochemistry 2004; vol. 48 issue 2 [Apr-Jun]: 129-134
T wo independent studies using reverse transcriptase-polymerase chain reaction (RT$P(R)$, capable of detecting a wide range of enteroviruses, including poliovirus, identified a $73 \%$ or $>80 \%$ incidence of enterovirus nucleic acid sequences in amyotrophic lateral sclerosis (ALS) cases vs a $0 \%$ or $4 \%$ incidence in controls, respectively (Woodall et al., 1994; Berger et al., 2000). Further recent studies have also shown similar sequences in Japanese ALS cases (Giraud et al., 2001). Viral sequences were similar from all three studies and were related to human coxsackie B/echoviruses, but not human coxsackievirus $A$, poliovirus or other picornaviruses infecting humans and other mammals (Woodall et al., 1994; Berger et al., 2000). Two other studies found no enterovirus sequences in ALS cases and controls (Swanson et al., 1995; Walker et al., 2001), and another, enterovirus sequences in ALS, other neurological diseases and controls (Muir et al., 1996).

Poliovirus specifically targets lower motor neurones in infectious poliomyeltis; in addition, clinical histories and histopathological comparisons with poliomyeltis suggest that enteroviruses may be implicated in ALS (Poskanzer et al., 1969; Roos et al., 1980). Polioviruses, extreme examples of acute lytic infective agents of the CNS, although not detected in ALS spinal cord, do exhibit some characteristics that illustrate how this group of viruses as a whole may be capable of acting as aetiologic agents in ALS. Poliovirus can enter the CNS through damaged motor end plates and track along nerves from the periphery to motor neurones, then spread within the motor system (Gromeier et al., 1998; Ohka et al., 1998; Ponuraj et al., 1998; Ponuraj et al., 2001 Strebel et al., 1995). Attenuated polioviruses might persist in motor neurones, but may not directly lyse them and induce only mild inflammation (Ponuraj et al., 2001) and poliovirus can be adapted to persist in mice motor neurones for more than 12 months (Destombes et al., 1997). RT-in situ-PCR located 
these sequences in the cytoplasm of neurone-like cells of the spinal cord (Berger et al., 2000). Two virus-sequence positive ALS cases repeatedly showed a very strong RT-PCR signal on RNA isolated from sequential wax sections in one study (Woodall et al., 1994). Adjacent wax sections to those analyzed by RT-PCR were therefore further analyzed by in situ hybridization, a technique which gives a higher resolution than RT-in situ-PCR, and here we present evidence for localization of enterovirus (EV) sequences to neurone-like cells in the spinal cord or lower medulla of these cases. A previous study had given an indication of the presence of enteroviruses in MND/ALS spinal cord by in situ hybridization (Brahic et al., 1985), but in this study we were able to enhance sensitivity by using highly viral sequence specific probes.

\section{Materials and Methods \\ Samples}

Cases used in this study were obtained from routine post-mortem material at two different sources in the West of Scotland between 1986 and 1988. Diagnosis of ALS was confirmed histopathologically in both cases (Woodall et al., 1994). Case \#1, at post mortem, was a female aged 83 showing a 12 month rapid progressive history of difficulty in swallowing and dysarthia, with limb fasciculation/weakness and loss of neurones in the anterior horn of the spinal cord and the hypoglossal nuclei. Case \#2 was a male aged 63 with a 60 month history of progressive muscle weakness, dysarthia, dyspnoea and increased tendon reflexes. Loss of neurones was recorded in the anterior horn of the spinal cord. The hypoglossal nuclei were not investigated. The control case was a 76 year old female who died from pneumonia and was autopsied 26 hrs after death.

Appropriate ethical approval had been obtained for this study.

\section{In situ hybridization}

Sections $(8.0 \mu \mathrm{m})$ from ALS cases \# 1 and 2 and a control spinal cord were dewaxed in xylene, rehydrated, then pretreated with $0.2 \mathrm{~N} \mathrm{HCl}$ for $10 \mathrm{~min}$ utes, $0.3 \% \mathrm{v} / \mathrm{v}$ triton $\mathrm{X}-100$ for $10 \mathrm{~min}, 10.0 \mathrm{mg} / \mathrm{mL}$ of proteinase $\mathrm{K}$ at $40^{\circ} \mathrm{C}$ for 15 minutes and postfixed in $4 \% \mathrm{w} / \mathrm{v}$ paraformaldehyde for 5 minutes. Sections were washed extensively after each of these procedures in PBS. One section was further treated with $20 \mathrm{mg} / \mathrm{mL}$ RNase A and $400 \mathrm{u}$ RNase Tl for 20 minutes at $37^{\circ} \mathrm{C}$, extensively washed, post-fixed in $4 \% \mathrm{w} / \mathrm{v}$ paraformaldehyde for 1 minute and again extensively washed. Sections were prehybridized in $2 \times \mathrm{SSPE} / 50 \%$ formamide at $37^{\circ} \mathrm{C}$ for 1 hour. RT-PCR products were amplified from the viral $5^{\prime}$ untranslated region of coxsackievirus B3, or from abelson cellular mRNA as previously described (Woodall et al 1994) and cloned directly into a transcription vector ( $p C R-S c r i p t$, Stratagene). Clone orientation was determined by sequencing analysis. Plasmids were double purified and either cleaved with Not-1 or EcoR1 (Roche), Qiagen tip purified and digoxigenin labelled sense and antisense riboprobes synthesized from either T7 or T3 RNA polymerases (20 u) for 2 hours (Roche). The reaction was terminated and the riboprobe/vector mix alkali treated at $60^{\circ} \mathrm{C}$ for 20 minutes, neutralized with sodium acetate buffer, $\mathrm{pH} 6.0$, then precipitated with the addition of $1 \mu \mathrm{L}$ of $20 \mathrm{mg} / \mathrm{mL}$ glycogen (Roche) and 2 volumes of ethanol. Riboprobes were dissolved in $100 \mu \mathrm{L}$ of RNase free water and $5.0 \mu \mathrm{L}$ checked by gel electrophoresis. A $6.0 \mu \mathrm{L}$ volume of riboprobe was added/100 $\mu \mathrm{L}$ hybridization mix/section (hybridization mix was $1 \mathrm{mM}$ Tris- $\mathrm{HCl}, \mathrm{pH} 7.0,1 x$ Denhardts solution, $2 \mathrm{x}$ SSPE, $50 \%$ v/v formamide, $0.5 \%$ SDS, $0.5 \%$ dextran sulphate, $0.25 \mathrm{mg} / \mathrm{mL}$ salmon sperm DNA). Separate adjacent sections were either probed with antisense riboprobes (detection of the + strand viral genome) or sense probes (negative control, but also will hybridize to the viral replicative intermediate). RNase pre-treated sections were probed with the anti-sense riboprobe. Riboprobes in hybridization mix, and prehybridise-mix treated sections, were incubated at $85^{\circ} \mathrm{C}$ for 2 minutes in parallel, then the riboprobe/hybridization mix applied to the sections and hybridized at $50^{\circ} \mathrm{C}$ overnight. Sections were washed in $2 \times \mathrm{SSPE}, 0.1 \times \mathrm{SSPE}$ for 10 minutes, $0.1 \times$ SSPE for 30 minutes at $50^{\circ} \mathrm{C}$ then $0.1 \times$ SSPE for 20 minutes. Standard colour development was performed - the digoxigenin antiserum was diluted $1 / 2000$ in $10 \%$ normal rat serum (Roche), and staining developed for 5 hours at $4^{\circ} \mathrm{C}$ in the dark. Sections were then washed in PBS, briefly counter-stained with eosin, washed in sterile distilled water, then mounted in Crystalmount (Biomeda, UK). Positively stained cells appeared as a deep purple against a light pink background. Negatively stained cells appeared pink, occasionally with a brown coloration. Differentially treated adjacent sections were matched for individual cells, or if this was not possible as a result of tissue damage or 

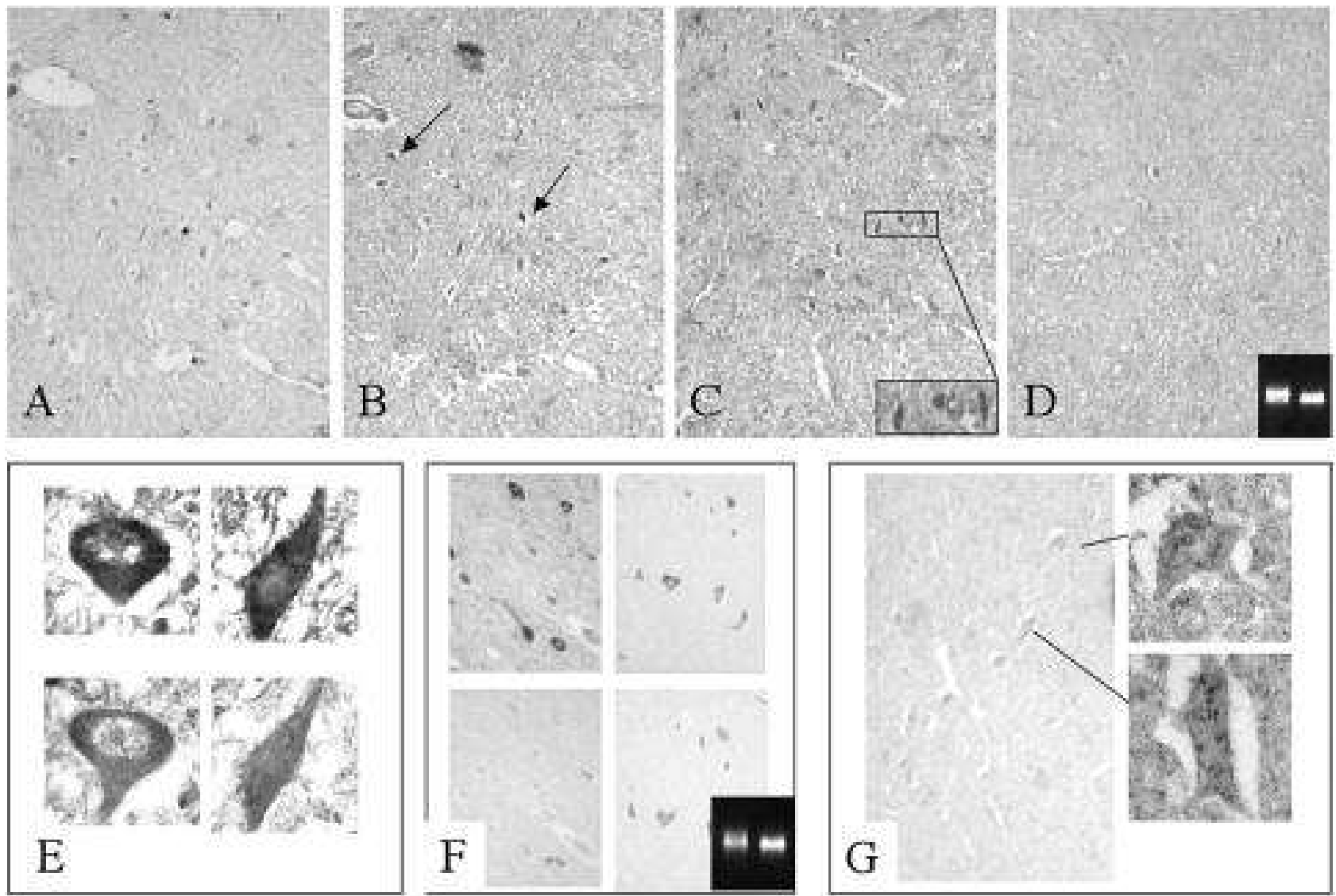

Figure 1. Cervical ventral horn of case 1. A. Right ventral horn from section stained with the coxsackievirus B sense (negative control) riboprobe (x40). B, Adjacent section showing matching neurones in the right ventral horn staining for the antisense coxsackivirus riboprobe (some of which are arrowed, $\mathrm{x} 40$ ). C. Left ventral horn showing staining of neurones with the coxsackivirus antisense riboprobe (x40). C inset. Enlargement (x200) showing stained ventral horn neurones. D. An adjacent section showing the left ventral horn staining for the antisense coxsackievirus riboprobe following treatment of the section with RNase. D (inset). Ethidium bromide stained gel showing enteroviral antisense (left) and sense (right) riboprobes. E. Matched ventral horn neurones stained with the coxsackievirus anti-sense probe (upper) and the sense riboprobe (lower). x1000 (oil immersion). F. Ventral horn neurones stained with protooncogene abelson anti-sense (left, upper) and sense probes (left, lower). Control spinal cord ventral horn neurons, antisense (right, upper) and sense (right, lower) staining for abelson (x200). F inset. Ethidium bromide stained gel showing abelson antisense(left) and sense (right) riboprobes. G. Ventral horn from a control spinal cord stained with the antisense riboprobe (x200). Selected neurones are shown $\mathbf{x 1 0 0 0 .}$

separation of sections, for the same region. Experimental and control photomicrographs, up to x400 magnification were taken under exactly the same manual exposure conditions using a Olympus $\mathrm{BH}-2$ microscope with an attached Olympus DP50 CCD camera. Images were digitized using AnalySIS 3.1 software (Soft imaging system, Gmbh). Photomicrographs at $\times 1000$ were taken with an oil immersion $x 100$ objective onto photographic slide film, then subsequently scanned into Adobe Photoshop 4.0 for Apple Macintosh.

\section{Results}

Staining of the cervical spinal cord of case \#1 is shown in Figure 1. The ventral horns of this case were considerably denuded of neurones, however remaining isolated neurones stained positive for the antisense viral riboprobe. Signal was located in neurones of both ventral horns (Figure $1 \mathrm{~B}, \mathrm{C}$ ) and was not observed in any other regions of the section. On an adjacent section the same ventral horn neurones were not stained by the sense (negative control) riboprobe (Figure $1 \mathrm{~A}$ ) and the antisense probe did not detect sequences in the anterior horn of a control spinal cord (Figure $1 \mathrm{G}$ ). Signal was not observed with the antisense riboprobe if a further adjacent section was RNase pre-treated (Figure 1 D). Examples of paired neurones stained with antisense or sense probes are shown (Figure 1 E). Signal for the mRNA of the cellular Abelson protein 


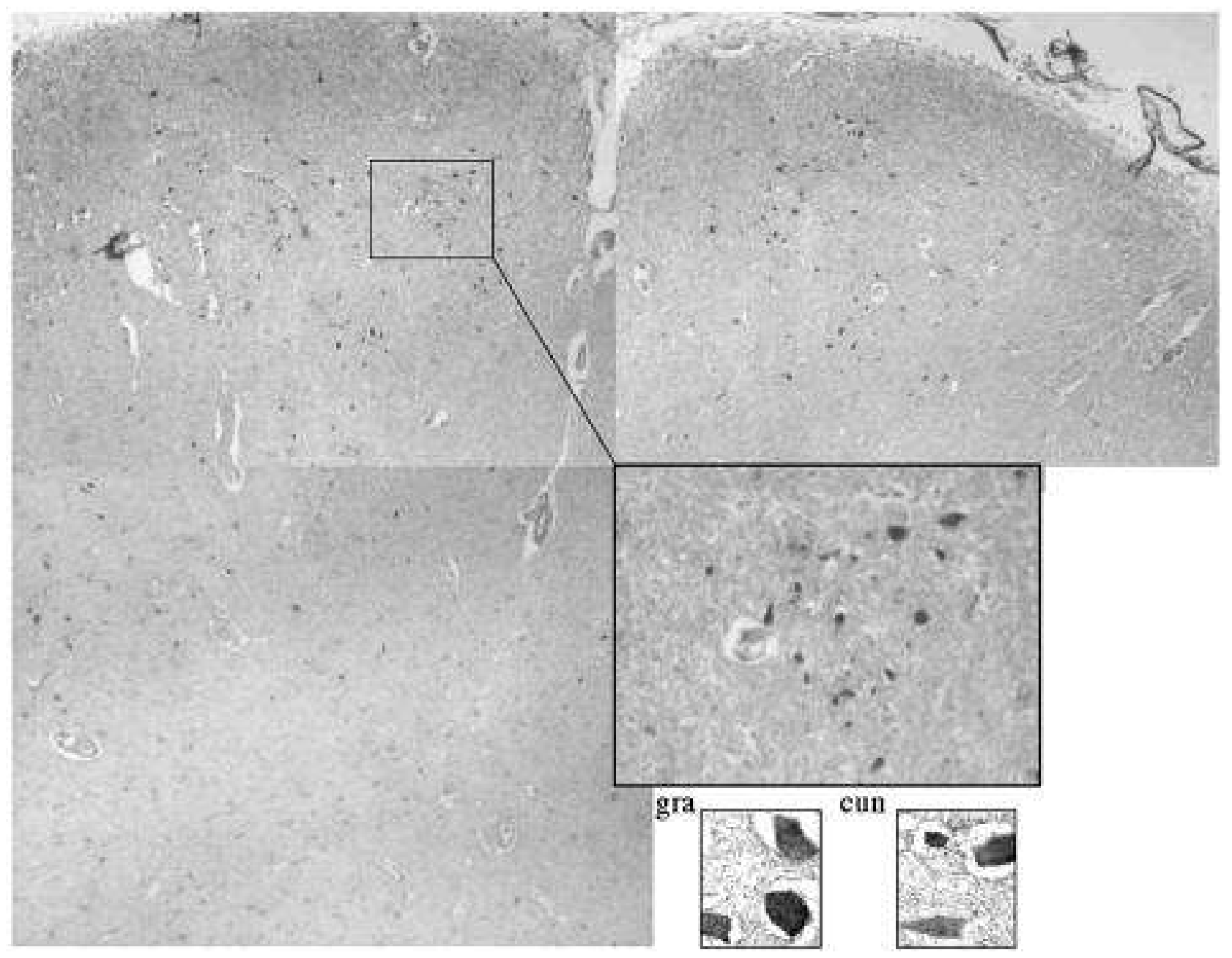

Figure 2. Case \#2. Composite image showing the distribution of signal in the gracile and cuneate nuclei in a section from the lower medulla (antisense coxsackievirus riboprobe $\times 40$ ). A selected cluster of stained neurones are shown x200. Stained (and unstained) neurones from the gracile (gra) and cuneate (cun) nuclei (x1000, oil immersion).

kinase was observed in neurones but not in other cell types, with the antisense, but not the sense riboprobe (Figure 1 F). Case \#2 showed a distinct pattern of staining in both the left and right gracile and cuneate nuclei, where many but not all neurones were stained (Figure 2). The staining pattern within these regions showed evidence of clustering, but there was not obvious perivascular staining. Staining was not observed in the gracile/cuneate nuclei of adjacent sections with the sense (negative control) riboprobe (Figure $3 \mathrm{~A}$ ) or $\mathrm{RNase}$ pre-treated sections stained with the anti-sense riboprobe (Figure 3B). Closer examination revealed stained neuronelike cells with a granular appearance in the gracile and cuneate nuclei (Figure 2). This staining was not observed with the sense riboprobe (Figure $3 \mathrm{~A}$, inset). Neurones showed faint labelling when stained with an abelson antisense, but not sense probe
(Figure 3C).

These data indicate that staining in both MND/ALS cases was virus-specific and localized to neurone-like cells. Ablation of signal from the antisense probe after RNase treatment, shows that RNA is specifically stained. Comparison of staining with sense and anti-sense probes, which were applied to adjacent sections in equal concentrations (see Figure 1D inset) shows that staining is specific to viral genome (+ sense) RNA. Not all neurones were labelled. The above evidence also suggests that this was not artefactual staining of neurones.

\section{Discussion}

The use of probes, the specificity of which was previously determined by sequencing RT-PCR products from adjacent sections, allows the highly sensi- 

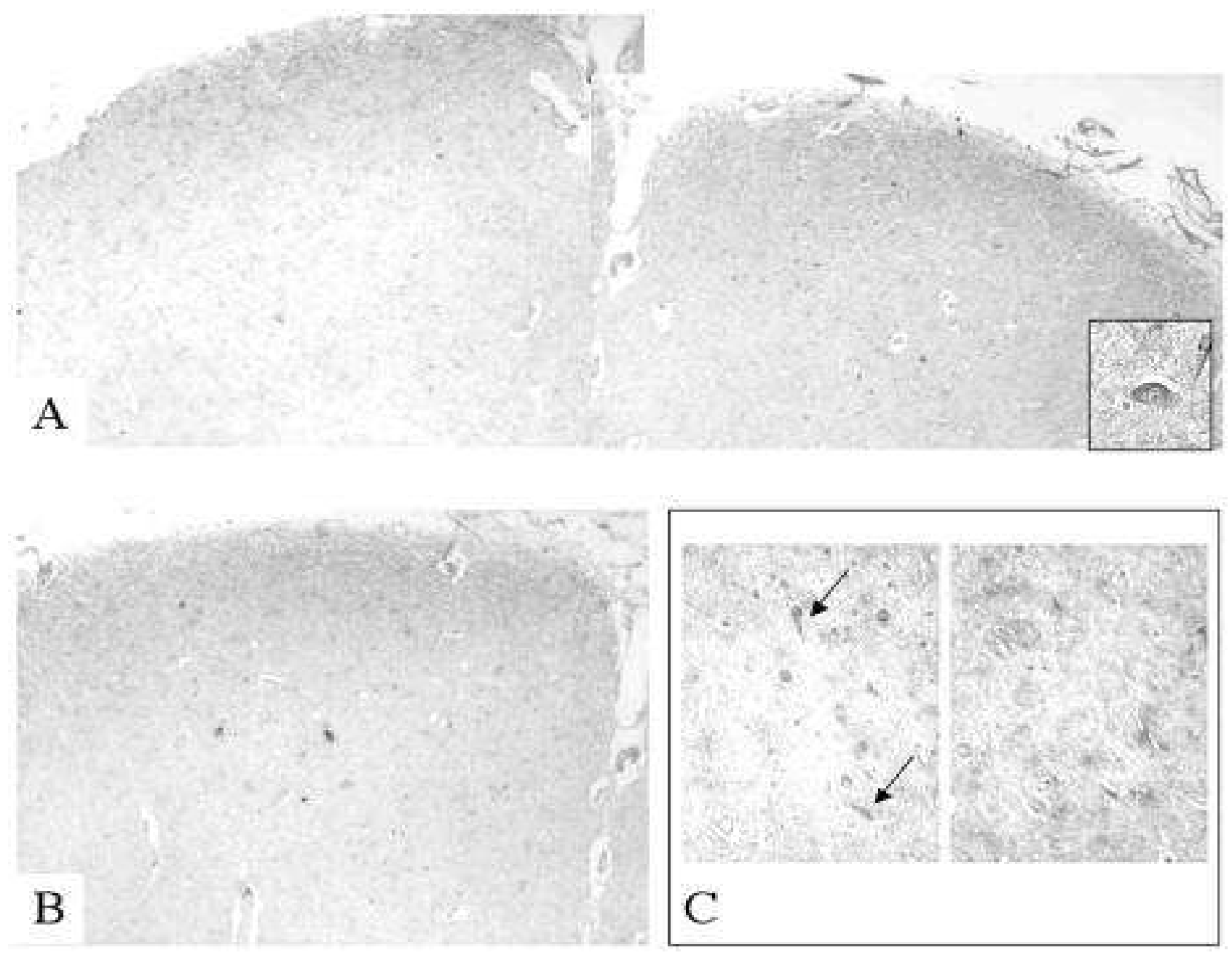

Figure 3. A. Case \#2. Composite image showing gracile/cuneate nuclei probed with the coxsackievirus B sense (negative control) riboprobe on sections adjacent to those probed with the antisense riboprobe in Figure 2 (x40). Inset. Neurone from this section (x1000, oil immersion). B. An adjacent section showing the right gracile and cuneate nuclei probed with the antisense coxsackievirus $B$ riboprobe following treatment with RNase $(x 40)$. C. Neurones probed with the proto-oncogene abelson antisense (left) and sense (right) riboprobes $(\mathbf{x} 200)$. Neurones are arrowed.

tive staining with digoxigenin-labelled riboprobes. Staining of cervical spinal neurones in case \#1 identified as motor neurones from their morphology and anatomical location in the ventral horn, is consistent with a role of enteroviruses in ALS but this is not evidence for an etiologic role. Examination of positively stained neurones indicates a cytoplasmic staining and a punctate appearance that is consistent with replication of enteroviruses (Bolten et al., 1998; Klinger et al., 2001). Many neurones in the gracile/cuneate nuclei of the lower medulla are stained for virus in case \#2. The gracile and cuneate nuclei are responsible for two point discrimination, vibration sense and conscious proprioception. Therefore, dysfunction of these neurones per se would not be expected to be responsible for the clinical signs of ALS. However, case \#2, in addition had shown loss of ventral horn motor neurones by rou- tine histopathological examination, indicative of ALS, but the hypoglossal nuclei had not been investigated. It is possible that loss of gracile/cuneate nuclei function is a secondary disease that could be masked by paralytic disease, especially if clinical manifestation occurred later in MND/ALS disease progression. The progression of this case was relatively slow (60 months), and this might have permitted wider dissemination of the virus into other systems from the motor system. Infection of the cuneate/gracile nuclei could originate from a persistent viral infection within the CNS by direct cell to cell contact similar to a mechanism observed in other picornaviruses (Wada et al., 1993). Notably, the gracile and cuneate nuclei lie adjacent to the hypoglossal nuclei in the brain stem, providing a possible opportunity for localized dissemination of virus from the motor system. Alternatively, this may 
be a widely disseminated acute infection, spread into the CNS by viraemia and is unrelated to MND/ALS. The lack of an observed inflammatory response, or obvious perivascular clustering of infected cells however, argues against this possibility, but does not exclude it. Further blocks from different levels of the spinal cord from case \#2 are available to us for further studies.

These viruses are incapable of latency, and therefore must be replicating in the cells they infect. They are committed to producing viral gene products that have potential to effect changes in cellular function and survival (Barco et al., 2000; Goldstaub et al., 2000; Huber et al.,1999; Iordanov et al., 2000; Teterina et al., 1997). This would support the hypothesis that the presence of these viruses indicates a contributory role in the pathogenesis of MND/ALS. Cases of MND/ALS, however, may be susceptible to opportunistic infection by enteroviruses. Motor end plate damage in infected atrophic striated muscle, for example, is known to stimulate entry into motor axons (Andrew et al., 1984; Gromeier et al., 1998; Sato et al., 1978), and therefore axonal withdrawal from muscle during motor neurone degeneration might provide an opportunity for enterovirus infection of the CNS.

\section{Acknowledgments}

We acknowledge the excellent technical support from Mary-Anne McKinnon. This work has been supported by the Motor Neurone Disease Association (UK) and the Scottish Motor Neurone Disease Association.

\section{References}

Andrew CG, Drachman DB, Pestronk A, Narayan 0. Susceptibility of skeletal muscle to coxsackie $A 2$ virus infection: Effects of botulinum toxin and denervation. Science 1984;223:714-6.

Barco A, Feduchi E, Carrasco L. Poliovirus protease $3 \mathrm{C}$ kills cells by apoptosis. Virology 2000;266:352-60.

Berger MM, Kopp N, Vital C. Detection and cellular localization of enterovirus RNA sequences in spinal cord of patients with ALS. Neurology 2000;54:20-5.

Bolten R, Egger D, Gosert R, Schaub G, Landmann L, Bienz K. Intracellular localization of poliovirus plus- and minus-strand RNA visualized by strand-specific fluorescent in situ hybridization. J Virol. 1998;72:8578-85.

Brahic M, Smith RA, Gibbs Jr CJ, Garruto RM, Tourtellotte WW, Cash $E$. Detection of picornavirus sequences in nervous tissue of amyotrophic lateral sclerosis and control patients. Annal Neurol 1985; 18:337-43.
Destombes J, Couderc T, Thiesson D, Girard S, Wilt SG, Blondel B. Persistent poliovirus infection in mouse motoneurons. J Virol 1997; 71:1621-8.

Giraud P, Beaulieux F, Ono S, Shimizu N, Chazot G, Lina B. Detection of enteroviral sequences from frozen spinal cord samples of Japanese ALS patients. Neurology 2001;56:1777-8.

Goldstaub D, Gradi A, Bercovitch Z, Grosmann Z, Nophar S, Sonenberg $\mathrm{N}$, Kahana C. Poliovirus $2 \mathrm{~A}$ protease induces apoptotic cell death. Mol Cell Biol 2000;20:1271-7.

Gromeier M, Wimmer E. Mechanism of injury-provoked poliomyelitis. J Virol 1998;72:5056-60.

Huber M, Watson KA, Selinka HC, Carthy CM, Klingel K, McManus $B M$, et al. Cleavage of RasGAP and phosphorylation of mitogenactivated protein kinase in the course of coxsackie B3 replication. J Virol 1999;73:3587-94.

Iordanov MS, Paranjape JM, Zhou AM, Wong J, Williams BRG, Meurs $E F$, et al. Activation of p38 mitogen-activated protein kinase and CJun NH2-terminal kinase by double-stranded RNA and encephalomyocarditis virus: Involvement of RNase $L$, protein kinase $\mathrm{R}$, and alternative pathways. Mol Cell Biol;20:617-27.

Klinger MHF, Kammerer R, Hornei B, Gauss-Muller V. Perinuclear accumulation of hepatitis $A$ virus proteins, RNA, and particles and ultrastructural alterations in infected cells. Arch Virol 2001; 146:2291-307.

Muir P, Nicholson F, Spencer G.T. et al. Enterovirus infection of the CNS of humans: lack of association with chronic neurological disease. J Gen Virol 1996;77:1469-76.

Ohka S, Yang W, Terada E, Iwasaki K, Nomoto A. Retrograde transport of intact poliovirus via the fast transport system. Virology 1998; 250:67-78.

Ponuraj EM, John TJ, Levin MJ, Simoes EAF. Cell to cell spread of poliovirus in the spinal cord of bonnet monkeys (Macaca radiata). J Gen Virol 1998;79:2393-403.

Ponnuraj EM, John TJ, Levin MJ, Simoes EA. Sabin attenuated $\mathrm{LSc} / 2 \mathrm{ab}$ strain of poliovirus spreads in the spinal cord from a peripheral nerve in bonnet monkeys (Macaca radiata). J Gen Virol 2001; 82:1329-38.

Poskanzer DC, Cantor HM, Kaplan GS. In: Norris, FH, Kurland, LT, eds Motor Neuron Diseases: Research in amyotrophic lateral sclerosis and related disorders., Grune and Stratton Inc, Philadelphia, 1969. p. 286-8.

Roos RPM, Viola MVM. Amyotrophic lateral sclerosis with antecedent poliomyelitis. Arch Neurol 1980;37:312-3.

Sato T, Chou SM. Effect of denervation on coxsackie A virus myositis in mice: an electronmicroscopic study. Neurology 1978;28:1232-40.

Strebel PM, Ion-Nedelcu N, Baughman AL, Sutter RW, Cochi SL. Intramuscular injections within 30 days of immunization with oral poliovirus vaccine - a risk factor for vaccine associated paralytic poliomyeltis. N Engl J Med 1995;332:500-6.

Swanson NR, Fox SA, Mastaglia FL. Search for persistent infection by poliovirus or other enteroviruses in amyotrophic lateral sclerosismotor neuron disease. Neuromuscul Disord 1995;5:457-65.

Teterina NL. Gorbalenya AE, Egger D. Bienz K, Ehrenfeld E. Poliovirus $2 \mathrm{C}$ protein determinants of membrane binding and rearrangements in mammalian cells. J Virol 1997;71:8962-72.

Wada YF. Viral-infection and dissemination through the olfactory pathway and the limbic system by Theilers virus. Am J Pathol 1993; 143: 221-9.

Walker MP, Schlaberg R, Hays AP, Bowser R, Lipkin WI. Absence of echovirus sequences in brain and spinal cord of amyotrophic lateral sclerosis patients. Ann Neurol 2001;49:249-53.

Woodall CJ, Riding MH, Graham DI, Clements GB. Sequences specific for enterovirus detected in spinal cord from patients with motor neurone disease. Br Med J 1994;308:1531-43. 